$$
\text { UNIVERSIDADE DE SÃO PAULO - USP }
$$

FACULDADE DE FILOSOFIA, CIÊNCIAS E LETRAS DE RIBEIRÃO PRETO MESTRADO EM ENTOMOLOGIA

RODRIGO ALVES DOS REIS

Diversidade de baratas (Blattodea: Blattaria) e de besouros bioluminescentes (Coleoptera:

Elateroidea: Elateridae: Agrypninae: Pyrophorini) em uma floresta estacional decidual 


\section{Diversidade de baratas (Blattodea: Blattaria) e de besouros bioluminescentes (Coleoptera: Elateroidea: Elateridae: Agrypninae: Pyrophorini) em uma floresta estacional decidual}

Dissertação apresentada à Faculdade de Filosofia Ciências e Letras de Ribeirão Preto da Universidade de São Paulo, como parte das exigências para a obtenção do título de Mestre em Ciências, obtido no Programa de PósGraduação em Entomologia.

Orientador: Prof. Dr. Jean Carlos Santos 


\section{FICHA CATALOGRÁFICA}

Autorizo a reprodução e divulgação total ou parcial deste trabalho, por qualquer meio convencional ou eletrônico, para fins de estudo e pesquisa, desde que citada a fonte.

Reis, Rodrigo Alves dos

Diversidade de baratas (Blattodea: Blattaria) e de besouros

bioluminescentes (Coleoptera: Elateroidea: Elateridae: Agrypninae:

Pyrophorini) em uma floresta estacional decidual, 2020.

34 p. : il. ; $30 \mathrm{~cm}$

Dissertação de Mestrado, apresentada à Faculdade de Filosofia Ciências e Letras de Ribeirão Preto/USP. Área de concentração: Entomologia.

Orientador: Santos, Jean Carlos.

1. Armadilha luminosa. 2. Blattodea. 3. Cerrado. 4. pirilampos. 


\section{Diversidade de baratas (Blattodea: Blattaria) e de besouros} bioluminescentes (Coleoptera: Elateroidea: Elateridae: Agrypninae: Pyrophorini) em uma floresta estacional decidual

Dissertação apresentada à Faculdade de Filosofia Ciências e Letras de Ribeirão Preto da Universidade de São Paulo, como parte das exigências para a obtenção do título de Mestre em Ciências, obtido no Programa de PósGraduação em Entomologia.

Aprovado em:

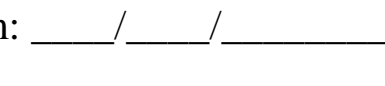

BANCA EXAMINADORA:

Prof. Dr. Jean Carlos Santos - Orientador

Universidade Federal de Sergipe (UFS)

Profa. Dra. Helena Maura Torezan Silingardi - Membro titular Universidade Federal de Uberlândia (UFU)

Profa. Dra. Simone Policena Rosa - Membro titular Universidade Federal de Itajubá (UNIFEI)

Profa. Dra. Sonia Maria Lopes Fraga - Membro titular Universidade Federal do Rio de Janeiro (UFRJ) 
Um haikai...

Só três frases...

Só uma fase... 


\section{Agradecimentos}

Primeiramente, agradeço ao ser mais poderoso do universo. Ele é tão grandioso que não mereço nem o professar neste trabalho.

Ao Instituto Estadual de Florestas (IEF), pela permissão concedida para trabalharmos no Parque Estadual do Pau Furado (PEPF), e ao Governo do Estado de Minas Gerais, por meu provimento.

Ao Prof. Dr. Jean Carlos Santos, meu orientador, meus sinceros agradecimentos pela mais diversa e infinita ajuda.

Aos meus colegas de laboratório que me ajudam (e ajudaram) muito. Sem eles, seria impossível realizar este feito: Henrique, sempre solícito; Fernanda, sempre sistemática; Afonso, sempre "de boa"; Guilherme, sempre atuante; Michele, sempre audaz; Marcela, sempre atenta; Lucas ("Chico"), sempre à disposição; Thais, sempre presente.

A todos os trabalhadores da Universidade de São Paulo (USP), da Universidade Federal de Uberlândia (UFU), da Universidade Federal de Itajubá (UNIFEI) e da Universidade Federal do Rio de Janeiro (UFRJ), que contribuíram direta e indiretamente para este trabalho.

À Profa. Dra. Cecília Lomônaco de Paula, por ter me iniciado ao meio acadêmico.

Ao Prof. Dr. Marcos Antônio Alves Carneiro, pela ajuda em momentos inóspitos.

À Profa. Dra. Helena Maura Torezan Silingardi, que me socorreu e me ajudou em meio à correria, e ao seu marido, Prof. Dr. Kleber Del Claro, que me ensinou a "defender" na hora - "não importa como, defenda respeitando o prazo". Que esta família continue ajudando vários docentes!

A todos os meus colegas de USP: à Renata Aparecida de Andrade Cavallari da secretaria e à Vera Cassia Cicilini de Lucca da secretaria da pós graduação em Biologia Comparada; ao Prof. Dr. Jimi Naoki Nakagima, que identificou as plantas coletadas; à Profa. Dra. Sonia Maria Lopes, da Universidade Federal do Rio de Janeiro que identificou Blattaria; à Profa. Dra. Simone Policena Rosa, da Universidade Federal de Itajubá (UNIFEI) que identificou Elateridae; e ao Prof. Dr. José Ricardo Miras Mermudes, da Universidade Federal do Rio de Janeiro que identificou Phengodidae e Lampyridae. Aos que ministraram aulas no curso de mestrado em Entomologia da USP: Prof. Dr. Rhayner Guilhermo Nascimento Ferreira, Profa. Dra. Vera Lúcia Imperatriz-Fonseca, Prof. Dr. Pitágoras da Conceição Bispo, Profa. Dra. Zilá Luz Paulino Simões, Prof. Dr. Fábio Santos do Nascimento, Prof. Dr. Dalton de Souza Amorin, Prof. Dr. Marcelo Tadeu Motokane, Prof. 
Dr. Jean Carlo Santos. Obrigado pelos ensinamentos nesta caminhada!

Aos colegas de trabalho da Escola Estadual do Parque São Jorge (EEPSJ):

Aos colegas de trabalho da Escola Estadual Professor José Ignácio de Sousa (EPJIS):

À minha família, pelo apoio.

À minha esposa...

À minha filha...

Ao meu filho...

E a todos os meus parentes (até os que já se foram) que me apoiaram e me ensinaram durante toda a minha vida. 


\section{Resumo}

Os estudos sobre a diversidade de baratas e besouros bioluminescentes são escassos em vários biomas, por exemplo, para o Cerrado. Este estudo teve como objetivo inventariar os gêneros de baratas (Blattodea: Blattaria) e as espécies de besouros bioluminescentes (Coleoptera: Elateroidea: Elateridae: Agrypninae: Pyrophorini) em uma floresta estacional decidual no Parque Estadual do Pau Furado localizado nos municípios de Uberlândia e Araguari no Estado de Minas Gerais, Brasil, e adicionalmente, comparar e analisar e a riqueza e abundância destes insetos entre dois estágios de conservação florestais (área conservada em comparação com área em regeneração). Desses insetos foram amostrados usando seis armadilhas luminosas modelo Luiz de Queiroz durante sete meses, entre outubro de 2018 e abril de 2019. Nove gêneros de baratas e sete espécies de Pyrophorini foram encontrados. As baratas foram mais abundantes no mês de outubro, enquanto para os pirilampos, este pico ocorreu em novembro. Os resultados mostraram que baratas foram mais comuns em ambientes em regeneração em detrimento de ambientes conservados. Enquanto para os pirilampos, a abundância não diferiu estatisticamente entre as áreas. As baratas podem ser importantes em ambientes em regeneração e os vagalumes podem estar presentes em vários habitats com um específico grau de tolerância à ação antrópica.

Palavras-chave: Armadilha luminosa, Blattodea, Cerrado, pirilampos. 


\begin{abstract}
Studies on the diversity of cockroaches and bioluminescent beetles are scarce in several biomes, for example, for the Cerrado. The aim of this study was to inventory the genera of cockroaches (Blattodea: Blattaria) and the species of bioluminescent beetles (Coleoptera: Elateroidea: Elateridae: Agrypninae: Pyrophorini) in a deciduous forest in the Parque Estadual do Pau Furado located in the municipalities of Uberlândia and Araguari in the State of Minas Gerais, Brazil, and additionally, to compare and to analyze and the richness and abundance of these insects between two stages of forest conservation (conserved area with in regeneration area). For this, the insects were sampled using six light traps model Luiz de Queiroz for seven months, between October 2018 and April 2019. Nine genera of cockroaches and seven species of Pyrophorini were found. Cockroaches were more abundant in October, while for fireflies peak occurred in November. The results showed that cockroaches were more common in regenerating environments to the detriment of conserved environments. While for fireflies, abundance did not differ statistically between areas. Cockroaches can be important in regenerating environments and fireflies can be present in various habitats with a specific degree of tolerance to anthropic action.
\end{abstract}

Key-words: Light trap, Blattodea, Cerrado, fireflies 


\section{Sumário}

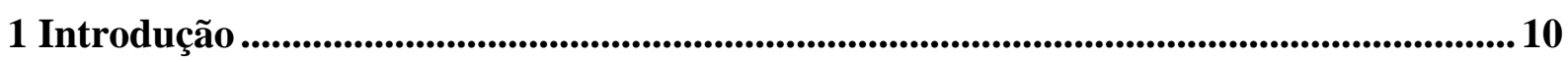

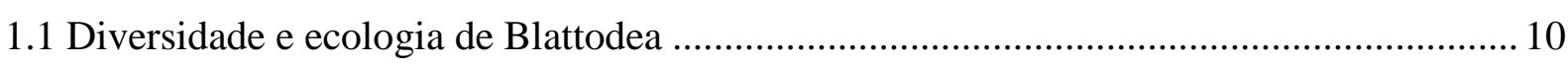

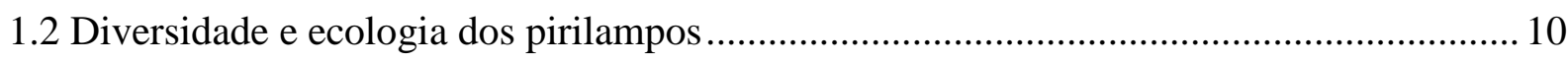

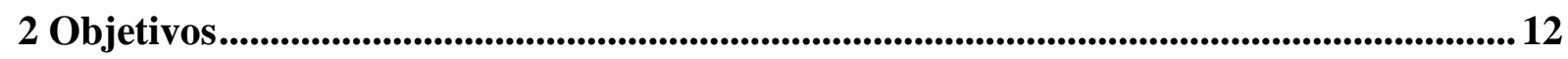

3 Material e métodos

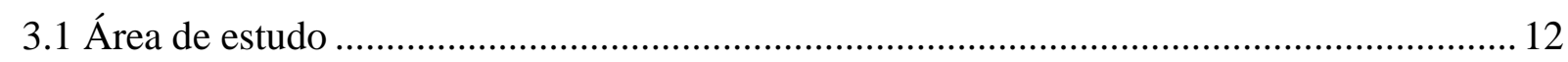

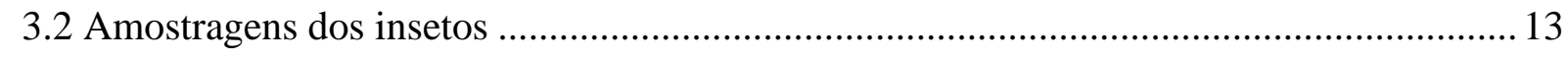

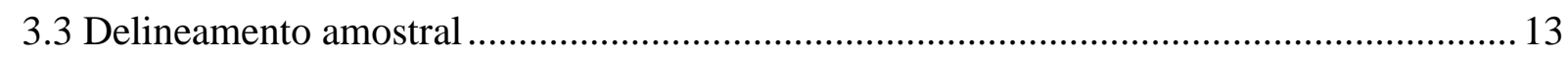

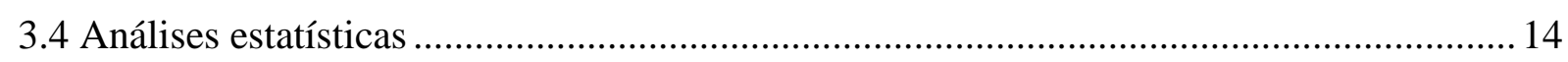

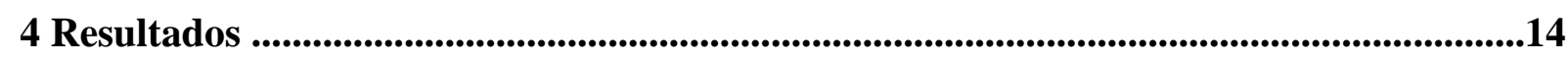

5 Discussão

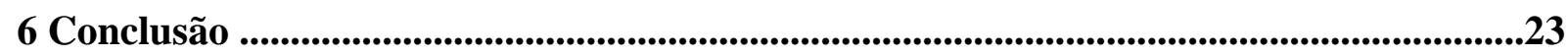

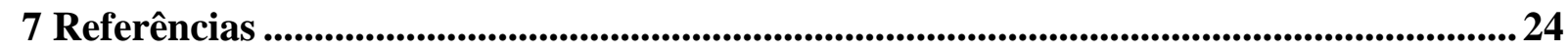

Declaração ........................................................................................................................................................ 28 


\section{Introdução}

\subsection{Diversidade e ecologia de Blattaria}

Dictyoptera é um dos grupos mais antigos do grupo dos insetos voadores (Robinson \& Robinson 2009) que inclui um ramo filogenético com mantódeos, isópteros e blatódeos (Klass \& Meier, 2006). Os dictiópteros constituem em um grupo monofilético (Grimaldi, 2001) por possuírem um ancestral em comum (Gaunt \& Miles, 2002; Deitz et al., 2003), e por causa da imensa semelhança na sequência de bases da proteína hemocianina (Pick et al., 2009). Dentre os Dictyoptera, a ordem Blattodea compreende os dois grupos filogeneticamente semelhantes: as baratas (Blattaria) e os cupins (Termitidae), sendo o último recentemente incluído nesse grupo (Inward et al., 2007; Harrison et al., 2018). Dentro de Blattodea, as baratas de destacam com cerca de 4.000 espécies descritas no mundo (Rafael et al., 2008), com cerca de 1.200 espécies na região neotropical (Buzzi \& Miyazaki, 2002), e 700 espécies no Brasil (Pellens \& Grandcolas, 2002).

As baratas são insetos sociais (Taucare-Ríos \& Faúndez, 2018), de corpos achatados e que podem atingir até $10 \mathrm{~cm}$ de comprimento (Pascual, 2015). As ninfas não têm asas, alguns adultos são alados e a maioria das espécies voam de maneira limitada. São omnívoras ou oligófagas, alimentando-se preferencialmente espécies vegetais. São ainda higrófilas e termófilas, adaptadas ao ambiente noturno, mas também são encontrados em refúgios escuros, como em frestas e cascas de árvores, folhiços (Beccaloni \& Eggleton, 2011), e ainda associadas à cupinzeiros, colmeias das abelhas (se alimentando das fezes), bromélias (de Oliveira et al, 1997), ninhos de aves (Di Iorio \& Turienzo, 2009) e ainda ocorrem em ecossistemas aquáticos (Lopes \& Oliveira, 2006; Lopes, 2015).

Algumas espécies de baratas, por exemplo Periplaneta americana (Linnaeus, 1758), estão adaptadas a ambientes antropizados com acúmulo de lixo ou esgoto (Rafael et al., 2008). Outras espécies são sinantrópicas, vivem em ambientes urbanos e degradados (Cruz Filho, 2017). Essas espécies podem ser vetores mecânicos de várias doenças e parasitoses humanas, sendo consideradas importantes no contexto médico-sanitário (Thyssen et al., 2004). No entanto, apesar de algumas espécies de baratas serem adaptadas aos ambientes antrópicos, a maioria das espécies podem ser sensíveis às alterações ambientais. Por exemplo, algumas espécies são encontradas em plantação de eucaliptos e matas, mas não são encontradas em plantações de cana de açúcar (de Fátima Martins et al., 2017).

\subsection{Diversidade e ecologia dos pirilampos}

A ordem Coleoptera Linnaeus, 1758 possui mais de 400 mil espécies descritas 
distribuídas em 211 famílias (Bouchard et al., 2011) e possui o maior número de espécies descritas dentre todas as ordens de insetos no mundo e no Brasil (Lewinsohn \& Prado, 2005). Os besouros se destacam em praticamente todos os ecossistemas terrestres e em alguns aquáticos, ao atuarem em vários níveis tróficos, como os necrófagos (decompositores), predadores e parasitas (Triplehorn \& Jonnson, 2011). Essa ordem é vital em vários serviços ecológicos, a exemplo da ciclagem de nutrientes; assim, espera-se que qualquer grau de alteração ou degradação no ambiente altera diretamente a abundância e a riqueza destes insetos. Dentre os besouros destaca-se a superfamília Elateroidea Lawrence, 1982, que possuem um pronoto alongado ântero-posteriormente, cinco segmentos tarsais, élitros com 9 ou 10 estrias, processo posternal alongado e larvas elateriformes (Kundrata et al., 2014). Os besouros Elateroidea abrangem as três famílias de besouros bioluminescentes: Phengodidae, Lampyridae e Elateridae.

As espécies de Elateridae Leach, 1815 são conhecidas popularmente como "tec-tec", e consistem de cerca de 200 espécies de besouros que possuem algum grau de bioluminescência (Bi et al., 2019). A bioluminescência nesta família é usada como atrativo sexual. As fêmeas adultas atraem os machos durante o voo nupcial. Assim, quanto mais intensa são as luzes, maior a chance da cópula ser bem-sucedida (Costa et al., 2010). As larvas são elateriformes (Farias, 2013) e a bioluminescência, quando presente pode ser usada para predar de outros invertebrados, por exemplo em cupinzeiros inativos ou com poucos cupins (Bechara \& Viviani, 2015).

Estudos realizados com Elateridae indicam que esse grupo pode ser usado como bioindicador de qualidade ambiental (Viviani, et al. 2010). A presença dos besouros bioluminescentes no céu à noite são um bom indicativo de qualidade ambiental e que estes habitats estão preservados, pois os besouros bioluminescentes não suportam grandes distúrbios antrópicos, tais como luzes artificiais. Estes besouros podem então ficar desnorteados e morrerem (Lloyd, 2006).

Dentre os Elateridae, os Pyrophorini são usados como bioindicadores em áreas em regeneração e nos processos de sucessão ecológica (Costa et al., 2010). A tribo Pyrophorini pertence à subfamília Agrypninae e se encontra praticamente em toda a América e na Austrália, com 144 espécies e 19 gêneros (Rosa, 2007). Os besouros dessa tribo se caracterizam pela fronte carenada e por esclerito ventral. A melhor forma de captura na fase adulta ocorre por meio de armadilhas luminosas. As larvas noturnas são predadoras e vivem no solo, dentro da madeira ou entre as rochas (Rosa, 2004; 2007). A fase larval é extremamente sensível e possui necessidades específicas em relação aos fatores abióticos 
(e.g., temperatura) e bióticos (e.g., quantidade de presas). Depois da fase pupal, a fase adulta são besouros noturnos, e herbívoros. Isto evita a competição intraespecífica, pela larva e adultos ocuparem nichos ecológicos diferentes. Mudanças nos padrões de comportamento e na abundância sazonal desses insetos têm sido utilizadas podem elucidar distúrbios ambientais em várias partes do mundo (Costa et al., 2010). Estudos com inventários desses Pyrophorini são utilizados para mensurar o ambiente em relação ao grau de conservação da área estudada (Viviani et al., 2010).

\section{Objetivos}

Este estudo teve como objetivo inventariar os gêneros de baratas (Blattodea: Blattaria) e as espécies de besouros bioluminescentes (Coleoptera: Elateroidea: Elateridae: Agrypninae: Pyrophorini) que habitam o Parque Estadual do Pau Furado e verificar se estes gêneros/espécies respondem às mudanças de vegetação, ao comparar a vegetação preservada (sem interferências antrópicas diretas) com a vegetação secundária (em processo de regeneração natural).

\section{Material e métodos}

\section{1 Área de estudo}

As coletas ocorreram no Parque Estadual do Pau Furado (PEPF), uma unidade de conservação estadual com 2.186 hectares e que está inserida no bioma Cerrado, localizada

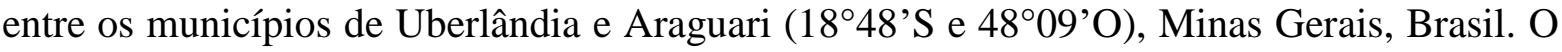
PEPF foi criado sob a forma de compensação do impacto ambiental causado pela represa de Capim Branco (Machado et al., 2007). A vegetação apresenta intercorrências com as formações florestais presentes no Vale do Rio Araguari (Rodrigues et al., 2010). O PEPF tem as seguintes fitofisionomias: floresta estacional semidecidual, floresta ciliar, floresta de galeria, vegetação secundária semidecídua, vegetação secundária ciliar, vegetação secundária cerradão, cerrado sentido restrito, cerradão, pastagem, cultura anual perene, floresta estacional decidual e vegetação secundária decídua. A floresta estacional decidual apresenta a maior área (1.118 hectares) e ocupa cerca de 50\% do parque, enquanto a vegetação secundária decídua, com 194 hectares, ocupa a segunda posição, com aproximadamente 9\% da área total. Antes de 2007, data da criação do Parque Estadual do Pau Furado, algumas áreas desta formação foram exploradas para a agricultura, pecuária e exploração seletiva de certas madeiras, e portanto, hoje, o PEPF tem áreas em regeneração que datam desde a referida criação do parque e áreas conservadas, pouco exploradas no passado. 


\subsection{Amostragens dos insetos}

Os besouros e as baratas foram amostrados usando uma armadilha luminosa, modelo Luiz de Queiroz. As armadilhas foram adquiridas da empresa BioControle ${ }^{\circledR}$ Ltda. e são feitas de armação de metal, com $69 \mathrm{~cm}$ de altura e $38 \mathrm{~cm}$ de largura. No centro de cada armadilha, havia uma lâmpada fluorescente ultravioleta (UV) de $15 \mathrm{~W}$ alimentada por bateria externa automotiva de 60 ampères. Um saco coletor de plástico de $60 \mathrm{~cm}$ foi acoplado embaixo da armadilha. Foram colocados dentro do saco vários papéis cortados para evitar o encontro dos insetos capturados e um pote de plástico com acetato de etila para matar os insetos. Cada armadilha possui um sensor de luminosidade que foi regulado para desligar as lâmpadas automaticamente. As armadilhas funcionaram no período noturno, entre 18:00h e 6:00h do outro dia. As coletas foram feitas por cinco noites consecutivas de lua nova, período escolhido por possuir menos luz natural. O pesquisador se apresentava no local na manhã seguinte para trocar o saco coletor contendo os insetos e verificar o funcionamento das armadilhas. Essas ações se repetiram mensalmente durante sete meses, entre outubro de 2018 e abril de 2019. No total, foram realizadas 2.520 horas de coleta noturna.

\subsection{Delineamento amostral}

Para a amostragem dos insetos, foram instaladas seis armadilhas luminosas em dois tipos de ambientes (três armadilhas na floresta estacional decidual e três armadilhas na vegetação secundária da floresta decídua - ambiente conservado versus ambiente antropofizado em regeneração), com distância mínima de 800 m entre elas. Após a coleta, os insetos foram triados, montados e organizados taxonomicamente conforme morfoespécies.

Em relação às baratas, essas foram acondicionadas em freezer a $-15^{\circ} \mathrm{C}$ e mantidas posteriormente em vidros com álcool a $70 \%$. Na sequência, as baratas foram enviadas ao Laboratório de Biodiversidade e Sistemática de Blattaria do Museu Nacional, mantido pela Universidade Federal do Rio de Janeiro (UFRJ), onde foram triadas, montadas em alfinetes entomológicos, e identificadas a nível de gênero com o auxílio de estereomicroscópio, e com base na literatura de Blattaria. As identificações foram feitas pela especialista no grupo, Profa. Dra. Sonia Maria Lopes.

Quanto aos besouros, esses foram primeiramente resfriados em freezer a $-15^{\circ} \mathrm{C}$ e, depois, em mantidos em álcool 96\%. Posteriormente, eles foram enviados e identificados com base na literatura de Pyrophorini pela especialista do grupo, Profa. Dra. Simone Policena Rosa, do Instituto de Recursos Naturais (IRN) da Universidade Federal de Itajubá 
(UNIFEI).

\subsection{Análises estatísticas}

Os dados foram analisados usando estatísticas descritivas. Não foi possível analisar os dados a nível de gênero e espécie, pois os conjuntos de dados ferem os pressupostos estatísticos. Para comparar o número de indivíduos de besouros e baratas entre áreas conservadas e regeneradas, os dados foram analisados usando Mann-Whitney. As análises foram feitas no programa Past 4.02 enquanto das figuras foram feitas usando Minitab ${ }^{\circledR}$ 17.1.0.

\section{Resultados}

\subsection{Baratas}

Foram encontrados 123 indivíduos de baratas pertencentes a nove gêneros, duas famílias e cinco subfamílias (Tabela 01). Chromatonotus foi o gênero com mais indivíduos $(\mathrm{N}=59)$, representando $48 \%$ do total de indivíduos amostrados. Entre os indivíduos capturados, em geral, machos $(\mathrm{N}=104)$ foram proporcionalmente mais frequentes que as fêmeas $(\mathrm{N}=19)$, numa razão sexual aproximadamente de 5:1.

Comparando os habitats amostrados dentro da floresta estacional decidual, nas áreas em regeneração foram encontrados 80 indivíduos (aproximadamente 65\%) pertencentes a nove gêneros, enquanto as áreas conservadas foram encontrados 43 indivíduos (aproximadamente 35\%) de cinco gêneros (Tabela 01). De fato, estatisticamente áreas regeneradas apresentarem quase o dobro de indivíduos em comparação com as áreas conservadas (Mann-Whitney $\mathrm{U}=90,5, p<0,05, \mathrm{~N}=36$, Figura 01). Isto também refletiu na presença e ausência dos gêneros nas áreas, sendo que, Anaplecta, Litoblatta, Lophoblatta e Panchlora não tiveram indivíduos amostrados nas áreas conservadas (Figura 02). Ao longo dos meses de amostragem, a abundância de baratas se mostrou assimétrica, com 71,54\% dos indivíduos amostrados em outubro, e diminuindo drasticamente entre dezembro e abril (Figura 03). 
Tabela 1: Gêneros de Blattaria amostrados em áreas em regeneração e conservadas de uma floresta estacional decidual do Parque Estadual do Pau Furado, Minas Gerais, Brasil. Os dados estão separados por sexo e os valores representam os números absolutos de indivíduos e entre parentesis, a porcentagem de indivíduos.

\begin{tabular}{|c|c|c|c|c|c|}
\hline \multirow[t]{2}{*}{ Famílias } & \multirow[t]{2}{*}{ Subfamílias } & \multirow[t]{2}{*}{ Gêneros } & \multirow[t]{2}{*}{ Sexo } & \multicolumn{2}{|c|}{ Número de indivíduos (\%) } \\
\hline & & & & Áreas em Regeneração & Áreas Conservadas \\
\hline \multirow[t]{2}{*}{ Blattoidea } & Anaplectidae & Anaplecta & q & $5(4,07 \%)$ & $0(0 \%)$ \\
\hline & & Anaplecta & $\hat{\sigma}$ & $2(1,63 \%)$ & $0(0 \%)$ \\
\hline \multirow[t]{6}{*}{ Blattellidae } & Blattellinae & Chromatonotus & 우 & $2(1,63 \%)$ & $0(0 \%)$ \\
\hline & & Chromatonotus & ๙ & $33(26,82 \%)$ & $26(21,13 \%)$ \\
\hline & & Ischnoptera & 우 & $2(1,63 \%)$ & $0(0 \%)$ \\
\hline & & Ischnoptera & o & $4(3,25 \%)$ & $3(2,44 \%)$ \\
\hline & & Litoblatta & q & $0(0 \%)$ & $0(0 \%)$ \\
\hline & & Litoblatta & $\hat{\sigma}$ & $2(1,63 \%)$ & $0(0 \%)$ \\
\hline \multirow[t]{2}{*}{ Blaberidae } & Epilamprinae & Epilampra & 우 & $0(0 \%)$ & $0(0 \%)$ \\
\hline & & Epilampra & o & $8(6,50 \%)$ & $2(1,63 \%)$ \\
\hline \multirow[t]{2}{*}{ Blaberidae } & Panchlorinae & Panchlora & 우 & $1(0,81 \%)$ & $0(0 \%)$ \\
\hline & & Panchlora & $0^{\pi}$ & $0(0 \%)$ & $0(0 \%)$ \\
\hline \multirow[t]{6}{*}{ Pseudophyllodromiidae } & Pseudophyllodromiinae & Amazonina & q & $2(1,63 \%)$ & $0(0 \%)$ \\
\hline & & Amazonina & o & $1(0,81 \%)$ & $2(1,63 \%)$ \\
\hline & & Cariblatta & 우 & $7(5,69 \%)$ & $0(0 \%)$ \\
\hline & & Cariblatta & o & $10(8,13 \%)$ & $10(8,13 \%)$ \\
\hline & & Lophoblatta & 우 & $0(0 \%)$ & $0(0 \%)$ \\
\hline & & Lophoblatta & o & $1(0,81 \%)$ & $0(0 \%)$ \\
\hline & & & TAL: & $80(65,04 \%)$ & $43(34,96 \%)$ \\
\hline
\end{tabular}




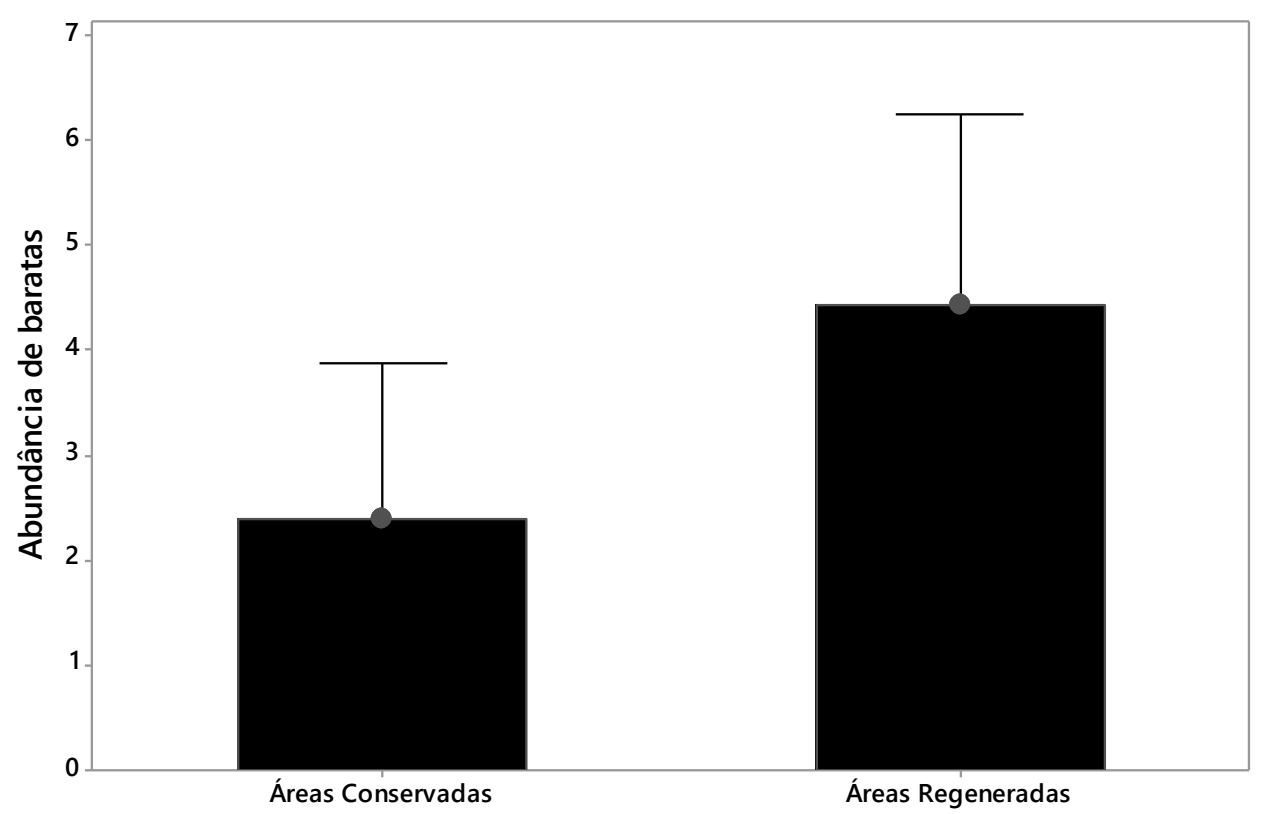

Figura 01. Média do número de indivíduos $( \pm \mathrm{EP})$ de Blattaria encontrados em áreas em regeneração e conservadas de uma floresta estacional decidual do Parque Estadual do Pau Furado, Minas Gerais, Brasil.

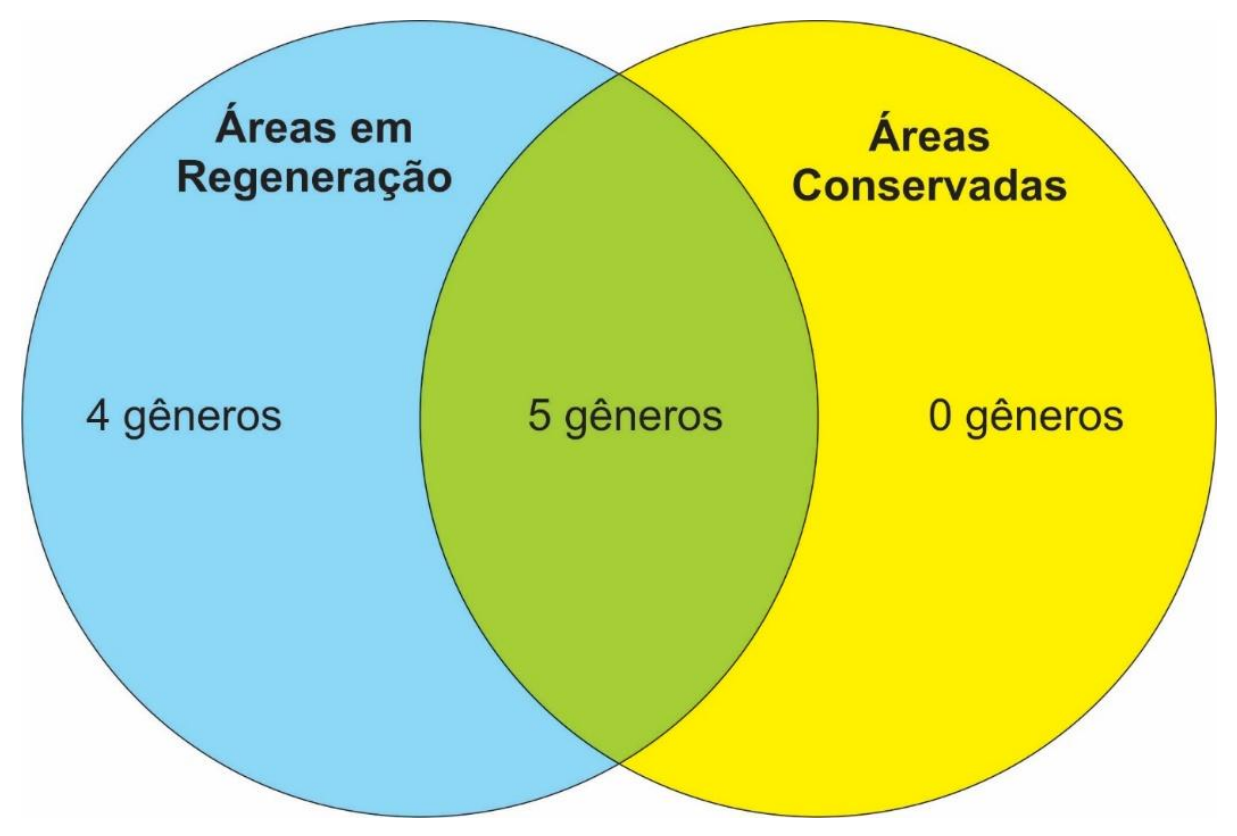

Figura 02. Diagrama de Venn do número de gêneros de Blattaria amostrados em áreas em regeneração e conservadas de uma floresta estacional decidual do Parque Estadual do Pau Furado, Minas Gerais, Brasil. 


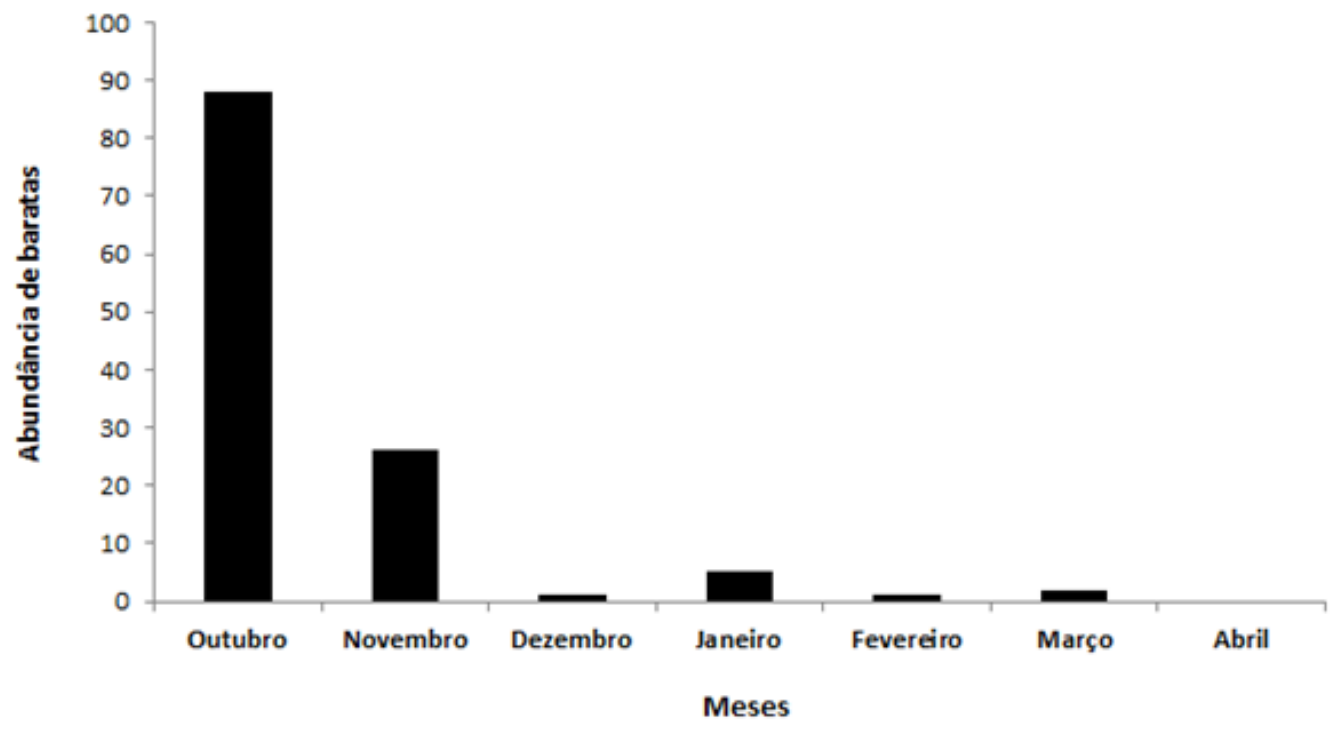

Figura 03: Distribuição do número de indivíduos de Blattaria amostrados no estudo ao longo dos meses em uma floresta estacional decidual do Parque Estadual do Pau Furado, Minas Gerais, Brasil.

\subsection{Besouros bioluminescentes}

Foram amostrados sete espécies de pirilampos pertencentes a quatro gêneros, são eles: Pyrophorus, Pyrearinus, Lygelater e Opselater. No total, foram amostrados 241 indivíduos, sendo as espécies Pyrearinus aff. castaneus (Costa, 1975) (N=120, 49,79\%), P. janus (Herbs, 1806) $(\mathrm{N}=84,34,89 \%)$ e Lygelater piceus (Schwarz, 1902) ( $\mathrm{N}=25,10,37 \%)$ as mais abundantes, representando aproximadamente $95 \%$ de todos os indivíduos amostrados (Tabela 02).

Comparando entre os habitats, nas áreas em regeneração foram encontrados 45 indivíduos $(18,67 \%)$ pertencentes a cinco espécies, enquanto as áreas conservadas foram encontrados 196 indivíduos $(81,33 \%)$ das sete espécies. Em relação às áreas conservadas, possivelmente a área $\mathrm{C} 1$ teve menos coleta de besouros devido a estar mais próximo a borda (Tabela 02). No entanto, apesar das áreas conservadas apresentarem quatro vezes mais besouros do que áreas regeneradas, a média de besouros não diferiu estatisticamente entre as áreas (Mann-Whitney U=20,5, p>0,05, N=14, Figura 04). Em relação às espécies, Opselater hebes (Germar, 1841) foi encontrada exclusivamente nas áreas em regeneração, enquanto Pyrophorus divergens (Eschscholtz, 1829) e P. pumilus (Candèze, 1863) foram encontradas exclusivamente nas áreas conservadas (Figura 05). Em relação à abundância entre os meses, o pico de coleta foi em novembro (59,34\% dos besouros coletados), seguido de outubro 
(35,27\% dos pirilampos coletados) e dezembro (5,39\% dos vagalumes coletados). Nos meses subsequentes, não foi coletado nenhum besouro bioluminescente (Figura 06).

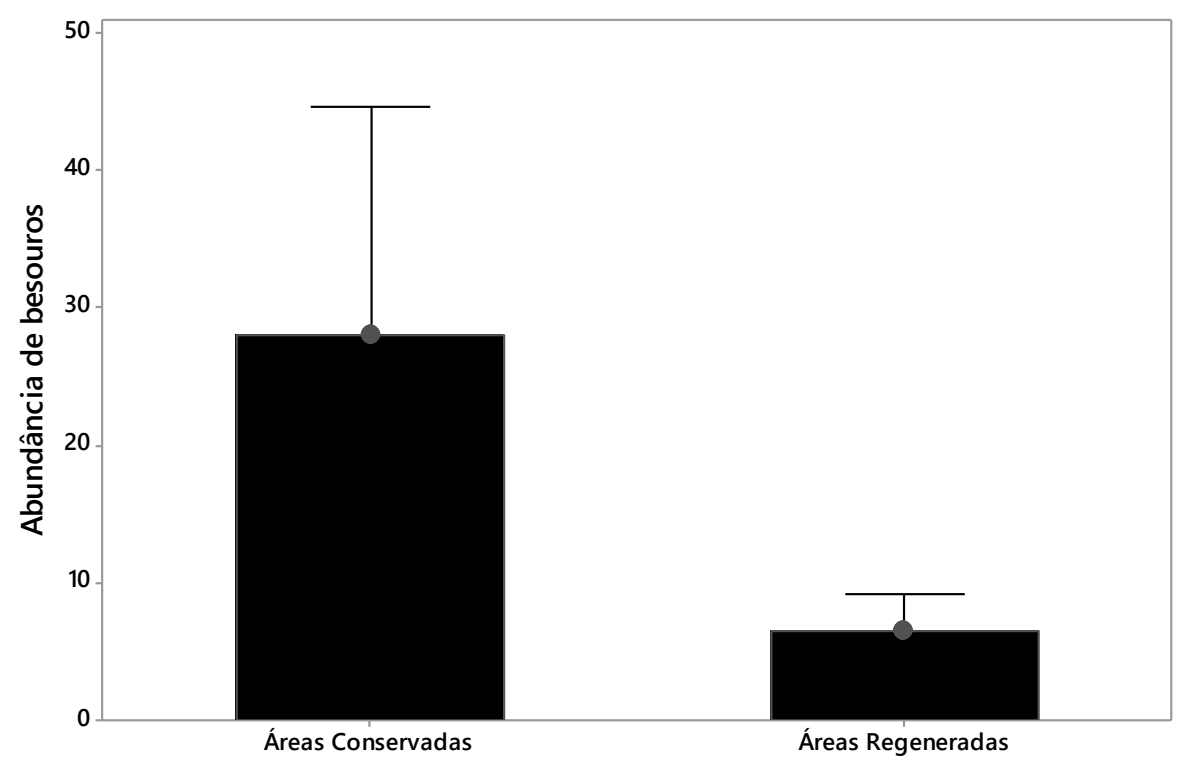

Figura 04: Média do número de indivíduos ( \pm EP) de besouros luminescentes (Coleoptera: Elateridae: Agrypninae: Pyrophorini) encontrado em áreas conservadas e regeneradas de uma floresta estacional decidual do Parque Estadual do Pau Furado, Minas Gerais, Brasil. 
Tabela 2: Espécies de besouros luminescentes (Coleoptera: Elateridae: Agrypninae: Pyrophorini) amostrados em áreas de regeneração e conservação de uma floresta estacional decidual do Parque Estadual do Pau Furado, Minas Gerais, Brasil. Os valores representam os números absolutos de indivíduos e entre parentesis, a porcentagem de indivíduos. Legenda: $\mathrm{C} 1$ - primeira área conservada, $\mathrm{C} 2$ - segunda área conservada, C3 - terceira área conservada, RC - área em regeneração perto da sede, RP área em regeneração perto da porteira, $\mathrm{RR}$ - área de regeneração perto do rio.

\begin{tabular}{|c|c|c|c|c|c|c|c|c|c|}
\hline \multirow{2}{*}{ Espécies } & \multicolumn{3}{|c|}{ Áreas em Regeneração } & \multicolumn{5}{|c|}{ Áreas Conservadas } & \multirow{2}{*}{ Total } \\
\hline & RC & $\mathbf{R P}$ & $\mathbf{R} \mathbf{R}$ & Subtotal & C1 & $\mathrm{C2}$ & $\mathbf{C 3}$ & Subtotal & \\
\hline Lygelater piceus (Schwarz, 1902) & $7(2,91 \%)$ & $4(1,66 \%)$ & $8(3,32 \%)$ & $19(7,89 \%)$ & $2(0,83 \%)$ & $3(1,24 \%)$ & $1(0,41 \%)$ & $6(2,48 \%)$ & $25(10,37 \%)$ \\
\hline Opselater hebes (Germar, 1841) & $2(0,83 \%)$ & $1(0,41 \%)$ & $0(0 \%)$ & $3(1,24 \%)$ & $0(0 \%)$ & $0(0 \%)$ & $0(0 \%)$ & $0(0 \%)$ & $3(1,24 \%)$ \\
\hline Pyrearinus aff. castaneus (Costa, 1975) & $4(1,66 \%)$ & $8(3,32 \%)$ & $0(0 \%)$ & $12(4,98 \%)$ & $11(4,56 \%)$ & $73(30,29 \%)$ & $24(9,96 \%)$ & $108(38,59 \%)$ & $120(49,79 \%)$ \\
\hline Pyrearinus janus (Herbs, 1806) & $10(4,15 \%)$ & $0(0 \%)$ & $0(0 \%)$ & $10(4,15 \%)$ & $16(6,65 \%)$ & $17(7,06 \%)$ & $41(17,03 \%)$ & $74(30,74 \%)$ & $84(34.89 \%)$ \\
\hline Pyrearinus pumilus (Candèze, 1863) & $0(0 \%)$ & $0(0 \%)$ & $0(0 \%)$ & $0(0 \%)$ & $2(0,83 \%)$ & $0(0 \%)$ & $3(1,24 \%)$ & $5(2,07 \%)$ & $5(2,07 \%)$ \\
\hline Pyrophorus divergens (Eschscholtz, 1829) & $0(0 \%)$ & $0(0 \%)$ & $0(0 \%)$ & $0(0 \%)$ & $1(0,41 \%)$ & $0(0 \%)$ & $1(0,41 \%)$ & $2(0,83 \%)$ & $2(0,83 \%)$ \\
\hline Pyrophorus tuberculifer (Eschscholtz, 1829) & $1(0,41 \%)$ & $0(0 \%)$ & $0(0 \%)$ & $1(0,41 \%)$ & $1(0,41 \%)$ & $0(0 \%)$ & $0(0 \%)$ & $1(0,41 \%)$ & $2(0,83 \%)$ \\
\hline Total & $24(9,96 \%)$ & $13(5,39 \%)$ & $8(3,32 \%)$ & $45(18,67 \%)$ & $33(13,69 \%)$ & $93(38,59 \%)$ & $70(29,05 \%)$ & $196(81,33 \%)$ & $241(100 \%)$ \\
\hline
\end{tabular}




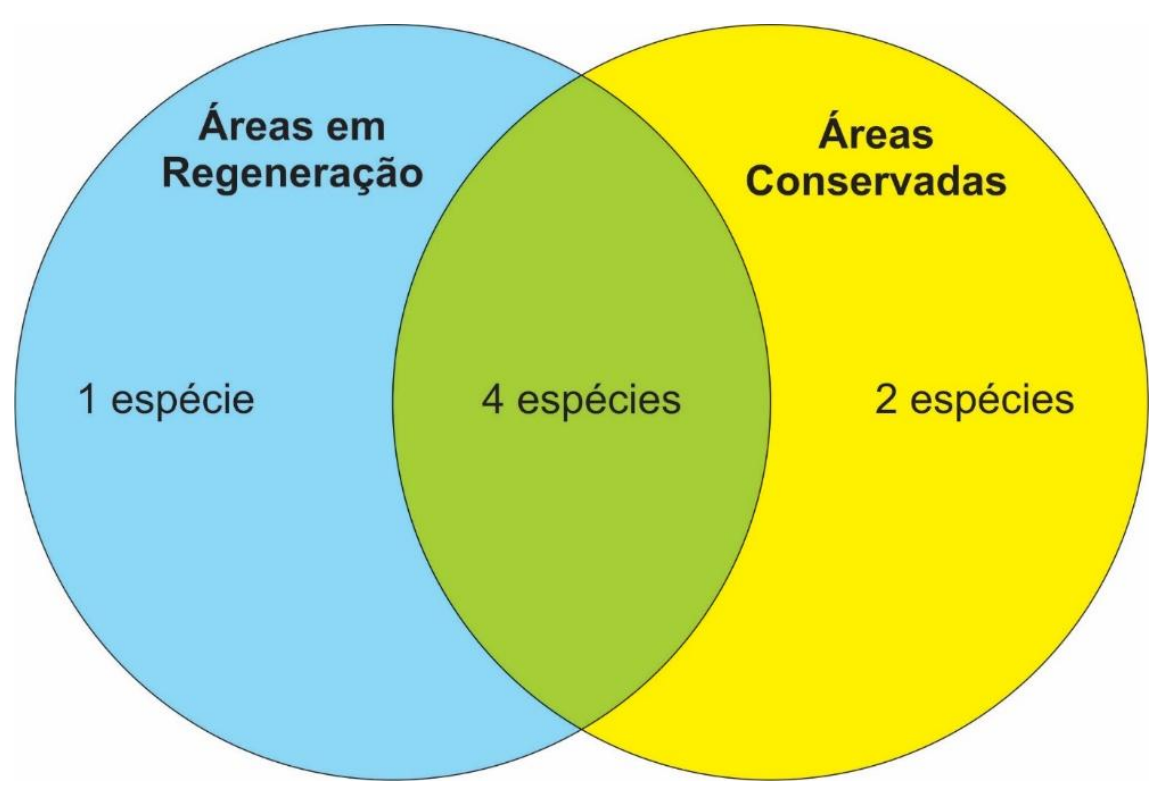

Figura 05: Diagrama de Venn do número de espécies de besouros luminescentes (Coleoptera: Elateridae: Agrypninae: Pyrophorini) amostrados em áreas de regeneração e conservação de uma floresta estacional decidual do Parque Estadual do Pau Furado, Minas Gerais, Brasil.

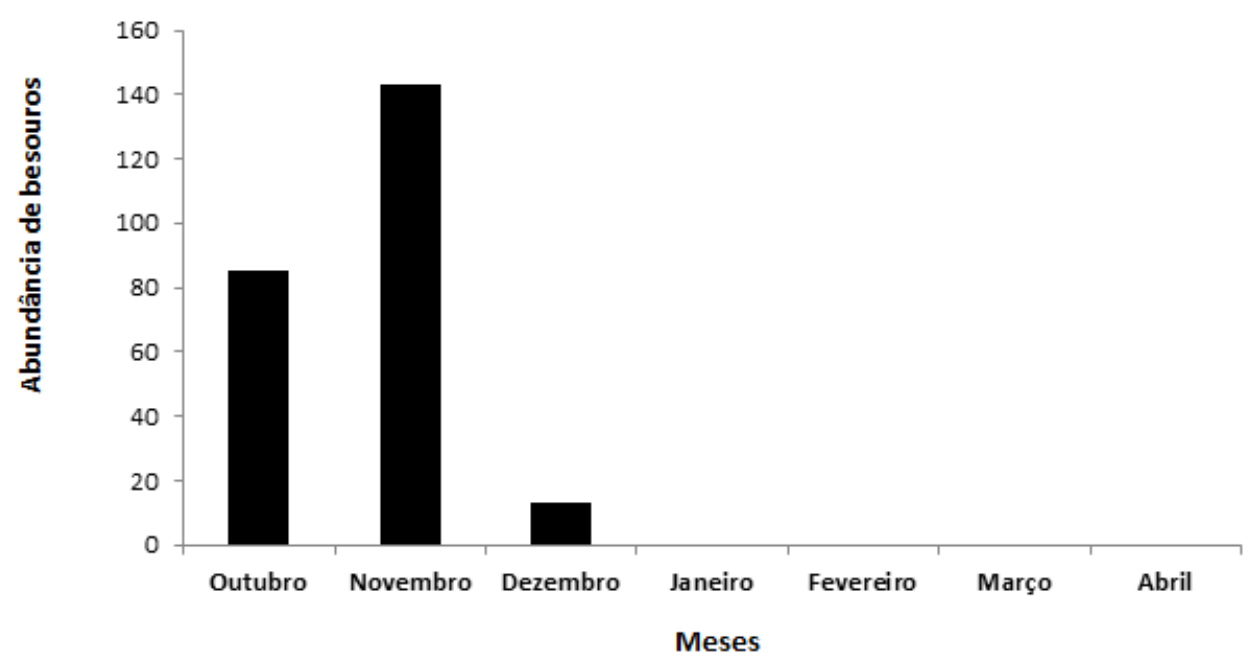

Figura 06: Gráfico de Barras representando o número de indivíduos de pirilampos amostrados no estudo ao longo dos meses em uma floresta estacional decidual do Parque Estadual do Pau Furado, Minas Gerais, Brasil. 


\section{Discussão}

\subsection{Baratas}

As baratas são organismos que podem contribuir para o processo sucessional de ecossistemas em regeneração. Algumas espécies de baratas são decompositoras, dentre as quais a mais comum é a barata doméstica (Periplaneta americana), que são bem específicas de ambientes degradados e/ou em regeneração (Rafael et al., 2008) e acabam contribuindo para ciclagem de nutrientes (Cole et al., 2016). Há também espécies específicas de ambientes conservados, como exemplo, alguns gêneros de baratas Eublaberus e Macrophyllodromia (Lopes \& de Oliveira, 2000; 2006).

Nossos resultados sugerem uma preferência por habitat, pois as baratas foram mais abundantes em ambientes em regeneração do que conservados. Isto indica que as baratas podem cumprir um papel importante em ambientes em regeneração, sendo vitais no processo de sucessão ecológica, pois elas podem ajudar na decomposição da matéria orgânica de ecossistemas sucessionais. Nossas sugestões convergem com os resultados de Silva et al., (2012), que demonstraram uma maior quantidade de baratas na borda, com mais estágios intermediários de sucessão, em detrimento de áreas do interior do fragmento ou do campo.

Os resultados também mostraram que houve um pico do número de indivíduos de Blattaria no início da estação chuvosa, em outubro. Neste período, há maior produtividade primária, o que reflete maior quantidade de recursos para os insetos (Luizão et al., 2014). Outros autores também encontraram maior quantidade de insetos em outubro, no período de transição da época seca para a chuvosa, por exemplo, em um ambiente de transição entre cerrado e caatinga em Minas Gerais (Silva, 2014) e no Cerrado de Brasília (Oliveira \& Frizzas 2008).

Os nove gêneros amostrados neste estudo foram comuns para o Cerrado e também para toda a região Neotropical (Pellens \& Grandcolas, 2008). Vale ressaltar que na Amazônia (Tarli, 2012) e na Mata Atlântica (Lopes, 2015) estão a maior quantidade de trabalhos publicados deste grupo no Brasil. Enquanto o Cerrado é um bioma pouco estudado e se conhece muito pouca das espécies de baratas. Portanto, é provável que futuramente novas espécies sejam descritas para este bioma (Lopes \& de Oliveira, 2000). Este estudo é um dos poucos que inventariou de baratas no Cerrado. Em um outro estudo de Oliveira \& Frizzas (2008), os autores que também usaram a armadilha luminosa Luiz de Queiroz para captura dos insetos no Cerrado do Distrito Federal, no entanto, os insetos amostrados foram identificados até ordem. O mérito desta dissertação foi identificar as baratas até gênero, mostrando quais destes gêneros habitam o cerrado. No futuro, ainda estes espécimes serão 
identificados até nível específico.

\subsection{Besouros bioluminescentes}

No Brasil, a maior parte dos estudos sobre inventário faunístico de pirilampos são dos estados de São Paulo (Viviani et al, 2010; Viviani \& Santos 2012; Santos et al., 2016) e do Rio de Janeiro (Corrêa et al., 2011) ambos estados supracitados no bioma de Mata Atlântica. Para o estado de Cerrado e para o estado de Minas Gerais este estudo é um dos pioneiros. Para nosso conhecimento, não há estudos que tratam exclusivamente sobre a diversidade de Pyrophorini para o Cerrado ou para o estado de Minas Gerais. No entanto, existem vários estudos a nível de ordem (Coleoptera) e, portanto, não se pode afirmar entre os espécimes amostrados haviam espécies de vagalumes (Oliveira \& Frizzas 2008).

Em relação às espécies amostradas nesse estudo, as sete espécies de Pyrophorini possuem uma ampla distribuição geográfica, basicamente por toda a região Neotropical (Costa et al., 2010). O gênero Lygelater apareceu em todas as armadilhas e áreas amostradas, sendo comprovada a sua vasta distribuição na américa neotropical no estudo de MartínezLuque (2014). Já as espécies Pyrophorus divergens Eschscholtz, 1829 e Pyrearinus pumilus Candèze, 1863 foram bem específicos para as áreas conservadas. De fato, estas espécies parecem estar relacionadas com ambientes preservados. Santos et al. (2016) encontraram $P$. divergens em ambientes conservados de fitofisionomia de Mata Atlântica do sudeste paulista. Pyrearinus pumilus carece de estudos, apenas recentemente foram estudados seus estágios larvais (Rosa et al., 2020; Viviani \& Amaral, 2016). Por fim, as outras espécies, Pyrearinus aff. castaneus Costa, 1975 e Pyrearinus janus Herbs, 1806, possuem uma distribuição geográfica maior, são mais tolerantes, e são encontradas também em áreas com um certo grau de perturbação (Costa, 2000).

Os pirilampos são bons indicadores ambientais, e este estudo sugere que algumas espécies aparentemente são sensíveis à degradação ambiental, enquanto outras espécies, mais tolerantes à degradação do habitat. Por exemplo, apenas uma espécie de pirilampo foi capturada em ambientes exclusivamente em regeneração (veja Tabela 02 e Figura 05). Provavelmente, isto reflete o estado de conservação da vegetação é um importante caracteristica para a presença e ausência das espécies de vagalumes (Viviani et al., 2010). Quando se observa os resultados, vê-se claramente que números espécies de pirilampos diferiram entre os ambientes em regeneração e conservados, sendo que ambientes 
conservados apresentariam mais espécies de Pyrophorini. No entanto, estes resultados não foram testados estatisticamente, indicando apenas uma tendência. Já a abundância dos besouros, esta não diferiu estatisticamente, provavelmente devido à baixa quantidade amostral e baixa quantidade de espécies amostradas.

\section{Conclusão}

Essa dissertação é a primeira a estudar a diversidade de gêneros de baratas (Blattaria) e de espécies de besouros bioluminescentes (Pyrophorini) em floresta estacional decidual e um dos poucos estudos envolvendo estes dois grupos para o Cerrado. Esta dissertação sugere que as baratas podem ser importantes componentes em ambientes em regeneração, e que as espécies de besouros bioluminescentes podem ser sensíveis ao tipo de ambiente e responderem à perda de habitat. No entanto, é importante estas conclusões devems er vistas com cautela, pois ambos os grupos estudados não tiveram um número de gêneros/espécies e/ou indivíduos suficientemente amostrados para se testar com eficiência as hipóteses levantadas aqui nesta dissertação. 


\section{Referências}

Beccaloni, G. W., \& Eggleton, P. (2011). Order Blattodea Brunner von Wattenwyl, 1882. In Z.-Q. Zhang (Ed.) Animal biodiversity: an outline of higher-level classification and survey of taxonomic richness. Zootaxa, 3148(1), 199-200.

Bechara, E. J., \& Viviani, V. R. (2015). Luzes vivas na escuridão: Fatos e Casos. Revista Virtual de Química, 7(1), 3-40.

Bi, W. X., He, J. W., Chen, C. C., Kundrata, R., \& Li, X. Y. (2019). Sinopyrophorinae, a new subfamily of Elateridae (Coleoptera, Elateroidea) with the first record of a luminous click beetle in Asia and evidence for multiple origins of bioluminescence in Elateridae. ZooKeys, 864, 79.

Bouchard, P., Bousquet, Y., Davies, A. E., Alonso-Zarazaga, M. A., Lawrence, J. F., Lyal, C. H., \& Smith, A. B. (2011). Family-group names in Coleoptera (Insecta). ZooKeys, 88,1 .

Buzzi, Z. J., \& Miyazaki, R. D. (2002). Entomologia Didática. Curitiba: Editora da UFPR.

Cole, R. J., Holl, K. D., Zahawi, R. A., Wickey, P., \& Townsend, A. R. (2016). Leaf litter arthropod responses to tropical forest restoration. Ecology and Evolution, 6(15), 51585168 .

Corrêa, V. A., Casari, S. A., \& Mermudes, J. R. M. (2011). Inventário de Elateridae (Coleoptera) de Vila Dois Rios, Ilha Grande, Angra Dos Reis, Rio de Janeiro. Biota Neotropica, 11(4), 291-297.

Costa, C. (1975). Systematics and evolution of the tribes Pyrophorini and Heligmini, with description of Campyloxeninae, new subfamily (Coleoptera, Elateridae). Arq. Zool. Bras. vol. 26; $n^{\circ} 2$; pp. 49-190.

Costa, C. (2000). Estado de conocimiento de los Coleoptera neotropicales. In Proyecto Iberoamericano de Biogeografía y Entomología Sistemática: PRIBES 2000: trabajos del ler taller iberoamericano de entomología sistemática (pp. 99-114). Madrid: Sociedad Entomológica Aragonesa.

Costa, C., Lawrence, J. F., \& Rosa, S. (2010). " 4.7. Elateridae Leach, 1815". In Coleoptera, Beetles, Volume 2, Morphology and Systematics (Elateroidea, Bostrichiformia, Cucujiformia partim). Berlin, Boston: De Gruyter. doi: https://doi.org/10.1515/9783110911213.75

Cruz Filho, E. F. D. (2017). Fauna de blatódeos em área de mata associados à Usina Termelétrica (Complexo Parnaíba III), Santo Antônio dos Lopes-MA, Brasil. Monografia de Graduação, Universidade Federal do Maranhão, São Luís, MA, Brasil.

de Fátima Martins, L., de Morais Pereira, J., Tonelli, M., \& Baretta, D. (2017). Composição da macrofauna do solo sob diferentes usos da terra (cana-de-açúcar, eucalipto e mata nativa) em Jacutinga (MG). Revista agrogeoambiental, 9(1).

de Oliveira, M. G. N., Da Rocha, C. F. D., \& Bagnall, T. (1997). The animal community associated with the tank bromeliad Neoregelia cruenta (R. Graham) L.B. Smith, 
Associated Animal Community. Revista Bromélia, Rio de Janeiro - RJ, 4, (2), 13-22.

Deitz, L. L., Nalepa, C., \& Klass, K.-D. (2003). Phylogeny of the Dictyoptera Re-examined (Insecta). Entomologische Abhandlungen, 61(1), 69-91. Recuperado em 10 maio, 2020, de

http://scholar.google.com/scholar?hl=en\&btnG=Search\&q=intitle:Phylogeny+of+the+D ictyoptera+Re-examined+(Insecta)\#0

Di Iorio, O., \& Turienzo, P. (2009). Insects found in birds' nests from the Neotropical Region (except Argentina) and immigrant species of Neotropical origin in the Nearctic Region. Zootaxa, 2187(1), 1-144.

Djernæs, M., Varadínová, Z. K., Kotyk, M., Eulitz, U., \& Klass, K. D. (2020). Phylogeny and life history evolution of Blaberoidea (Blattodea). Arthropod Systematics \& Phylogeny, 78, 29-67.

Farias, P. R. S. (2013). Manual de entomologia geral. Belém: Editora da Ufra.

Gaunt, Michael \& Miles, Michael. (2002). An Insect Molecular Clock Dates the Origin of the Insects and Accords with Paleontological and Biogeographic Landmarks. Molecular biology and evolution. 19. 748-61. 10.1093/oxfordjournals.molbev.a004133.

Grimaldi, D. (2001). Insect evolutionary history from Handlirsch to Hennig, and beyond. Journal of Paleontology, 75(6), 1152-1160.

Harrison, M. C., Jongepier, E., Robertson, H. M., Arning, N., Bitard-Feildel, T., Chao, H., \& Gowin, J. (2018). Hemimetabolous genomes reveal molecular basis of termite eusociality. Nature ecology \& evolution, 2(3), 557-566.

Inward, D., Beccaloni, G., \& Eggleton, P. (2007). Death of an order: a comprehensive molecular phylogenetic study confirms that termites are eusocial cockroaches. Biology Letters, 3(3), 331-335.

Klass, K.-D., \& Meier, R. (2006). A phylogenetic analysis of Dictyoptera (Insecta) based on morphological characters. Entomologische Abhandlungen, 63(1-2), 3-50. Recuperado em 12 maio, 2020, de http://www.arthropod-systematics.de/EA_63_1-2/EntAbh63_12_KlassMeier3-50.pdf

Kundrata, R., Bocakova, M., \& Bocak, L. (2014). The comprehensive phylogeny of the superfamily Elateroidea (Coleoptera: Elateriformia). Molecular Phylogenetics and Evolution, 76, 162-171.

Lewinsohn, T. M., \& Prado, P. I. (2005). Quantas espécies há no Brasil. Megadiversidade, 1(1), 36-42.

Lloyd, J. E. (2006). Stray light, fireflies, and fireflyers. In Rich, C., \& Longcore, T. (Eds.). Ecological Consequences of Artificial Night Lighting (pp. 345-364). Whashington: Island Press.

Lopes, S. M., \& de Oliveira, E. H. (2000). Espécie nova de Eublaberus Hebard, 1919 do estado de Goiás, Brasil e notas sobre E. marajoara Rocha e Silva-Albuquerque, 1972 (Blaberidae, Blaberinae). Museu Nacional. 
Lopes, S. M., \& de Oliveira, E. H. D. (2006). Duas espécies novas de Macrophyllodromia do Estado do Acre, Brasil (Blattaria, Blattellidae) coletadas em ninhos de vespas. Iheringia. Série Zoologia, 96(2), 257-260.

Lopes, S. M. (2015). Baratas (Blattodea) da Reserva Biológica de Pedra Talhada. In A. Studer, L. Nusbaumer, \& R. Spichiger (Eds.). Biodiversidade da Reserva Biológica de Pedra Talhada (Alagoas, Pernambuco - Brasil). Boissiera, 68, 237-241.

Luizão, F., Pashanasi-Amasifuen, B., Del Castillo-Torres, D., \& Lavelle, P. (2014). Influência da massa e nutrientes da liteira sobre a composição dos macro-invertebrados em plantíos florestais na Amazônia peruana. Folia Amazónica, 23(2), 171-186.

Machado, M. P., Oliveira, D. A., \& Rosolen, V. S. (2007). Realidades e desafios da criação do Parque Estadual do Pau Furado enquanto medida compensatória da criação das Usinas Capim Branco em Uberlândia-MG. Simpósio Nacional de Geomorfologia, Universidade Federal de Viçosa, Viçosa, MG, Brasil.

Martínez-Luque, Erick. (2014). Estudio faunístico de la familia Elateridae (Insecta: Coleoptera) en la estación en biología Chamela, Jalisco, México DF. 10.13140/RG.2.2.33534.08000.

Oliveira, C. M., \& Frizzas, M. R. (2008). Insetos de Cerrado: distribuição estacional e abundância. Brasília: Embrapa Cerrados.

Pascual, F. 2015. Orden Blattodea. Revista IDE@-SEA, 48, 1-13. Recuperado em 21 maio 2020, de http://www.sea-entomologia.org/IDE@/revista_48.pdf

Pellens, R., \& Grandcolas, P. (2002). Are successful colonizers necessarily invasive species? The case of the so-called invading parthenogenetic cockroach, Pycnoscelus surinamensis, in the Brazilian Atlantic forest. Revue d'écologie, vol. 57, 253-261.

Pellens, R., \& Grandcolas, P. (2008). Catalogue of blattaria (insecta) from Brazil. Zootaxa, 1709(1), 1-109.

Pick, C., Schneuer, M., \& Burmester, T. (2009). The occurrence of hemocyanin in Hexapoda. FEBS Journal, 276(7), 1930-1941. doi: 10.1111/j.1742-4658.2009.06918.x

Rafael, J. A., Silva, N. M., \& Dias, R. M. N. S. (2008). Baratas (Insecta, Blattaria) sinantrópicas na cidade de Manaus, Amazonas, Brasil. Acta Amazonica, 38(1), 173178. doi: 10.1590/S0044-59672008000100020.

Robinson, W. H., \& Robinson, W. H. (2009). Blattaria. In Robinson, W. H. Handbook of Urban Insects and Arachnids (pp. 35-64). Cambridge: Cambridge University Press.

Rodrigues, V. H. P., Lopes, S. D. F., Araújo, G. D., \& Schiavini, I. (2010). Composição, estrutura e aspéctos ecológicos da floresta ciliar do Rio Araguari no Triângulo Mineiro. Hoehnea, 37(1), 87-105.

Rosa, S. P. (2004). Revisão do gênero Opselater Costa (Coleoptera, Elateridae, Agrypninae). Revista Brasileira de Entomologia, 48(2), 203-219.

Rosa, S. P. (2007). Análise filogenética e revisão taxonômica da tribo Pyrophorini Candeze, 
1863 (Coleoptera, Elateridae, Agrypninae). Tese de Doutorado, Universidade de São Paulo, São Paulo, SP, Brasil.

Rosa, S. P., Mariano, R. D. R., Viviani, V. R., \& Costa, C. (2020). Morphology of immature stages of Pyrearinus pumilus (Candèze, 1863 (Coleoptera: Elateridae: Pyrophorini): the click beetle responsible for the luminous canga caves in the state of Pará, Brazil. Zootaxa, 4778(3), 561-570.

Santos, R. M., Schlindwein, M. N., \& Viviani, V. R. (2016). Levantamento de Coleópteros bioluminescentes na Mata Atlântica da Serra da Paranapiacaba. Biota Neotropica, 16(1).

Silva, J. F., Barrabarra, A. J., Hoppen, C., \& Rocha, A. C. P. (2012) Estudo comparativo entre as ordens de insetos em ambientes diversos no Refúgio de Vida Silvestre dos Campos de Palmas. IV Contextos e Conceitos - Mostra de Produção Científica e Extensão Instituto Federal do Paraná - Campus Palmas.

Silva, J. D. O. (2014). Variação na diversidade de insetos e herbivoria entre grupos fenológicos de uma floresta tropical seca. Tese de Doutorado em Ecologia Universidade de Brasília, Brasília D.F.

Taucare-Ríos, A., Carvajal, M., \& Faúndez, E. I. (2018). Primer registro de Pycnoscelus surinamensis (Linnaeus, 1758)(Blattodea: Blaberidae) en Chile Continental. Boletín de la Sociedad Entomológica Aragonesa (SEA), 63, 240-242.

Tarli, V. D. (2012). Influência de fatores ambientais sobre a composição de espécies de baratas (Insecta: Blattaria) na Reserva Ducke, Manaus, Amazonas, Brasil.

Triplehorn, C. A., \& Johnson, N. F. (2011). Estudo dos insetos. São Paulo: Cengage Learning.

Thyssen, P. J., Moretti, T. D. C., Ueta, M. T., \& Ribeiro, O. B. (2004). O papel de insetos (Blattodea, Diptera e Hymenoptera) como possíveis vetores mecânicos de helmintos em ambiente domiciliar e peridomiciliar. Cadernos de Saúde Pública, 20, 1096-1102.

Viviani, V. R., Rocha, M. Y., \& Hagen, O. (2010). Fauna de besouros bioluminescentes (Coleoptera: Elateroidea: Lampyridae; Phengodidae, Elateridae) nos municípios de Campinas, Sorocaba-Votorantim e Rio Claro-Limeira (SP, Brasil): biodiversidade e influência da urbanização. Biota Neotropica, 10(2), 103-116.

Viviani, V. R., \& Santos, R. M. D. (2012). Bioluminescent Coleoptera of Biological Station of Boracéia (Salesópolis, SP, Brazil): diversity, bioluminescence and habitat distribution. Biota Neotropica, 12(3), 21-34.

Viviani, V. R., \& Amaral, D. T. (2016). First report of Pyrearinus larvae (Coleoptera: Elateridae) in clayish canga caves and luminous termite mounds in the Amazon Forest with a preliminary molecular-based phylogenetic analysis of the $\mathrm{P}$. pumilus group. Annals of the Entomological Society of America, 109(4), 534-541. 


\section{Declaração}

Declaro, para os devidos fins, que esta dissertação de mestrado, apresentada ao Programa de Pós-graduação em Entomologia da Faculdade de Filosofia, Ciências e Letras de Ribeirão Preto, da Universidade de São Paulo, com 0\% de similaridade com outros estudos, segundo o “Antiplagiarist” versão 2.7, um software farejador de plágio.

Uberlândia, 10 de setembro de 2020.

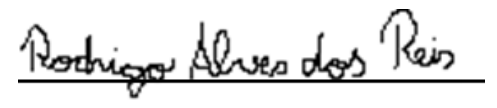

Rodrigo Alves dos Reis 